\title{
Novel TFAP2A mutation in a Japanese family with Branchio-oculo-facial syndrome
}

\author{
Taisuke Sato 10 1,2, Osamu Samura ${ }^{1}$, Noriko Kato ${ }^{2}$, Kosuke Taniguchi ${ }^{2}$, Ken Takahashi ${ }^{1,2}$, Yuki Ito ${ }^{1,2}$, Hiroaki Aoki ${ }^{1}$, \\ Masahisa Kobayashi ${ }^{3}$, Ohsuke Migita ${ }^{2}$, Aikou Okamoto ${ }^{1}$ and Kenichiro Hata ${ }^{2}$
}

\begin{abstract}
Branchio-oculo-facial syndrome (BOFS) is a rare autosomal dominant disorder characterized by craniofacial, ocular, and ectodermal anomalies. BOFS is caused by mutation of the transcription factor AP2-alpha gene (TFAP2A). We performed detailed genetic analysis of a Japanese family with clinically suspected BOFS and identified a novel missense mutation resulting in a predicted amino-acid substitution in the highly conserved basic DNA-binding domain of TFAP2A (NM_003220.2:c.699A>C).
\end{abstract}

Branchio-oculo-facial syndrome (BOFS, OMIM number: 113620) is a rare autosomal dominant inheritable condition that predominantly affects the development of the first and second pharyngeal arches. It is characterized by craniofacial, ocular, and ectodermal malformations but shows high variability in severity and clinical phenotype, including the expression of specific malformations ${ }^{1}$. One-third of patients with BOFS also exhibit renal system anomalies ${ }^{1}$. Transcription factor AP2-alpha (TFAP2A, OMIM number: 107580) is known to be the gene responsible for the development of BOFS, and specific TFAP2A functions have been elucidated in the affected tissues of patients with $\mathrm{BOFS}^{2-5}$. In the current report, we describe a family that has multiple anomalies that are clinically consistent with the BOFS spectrum and present results from genetic analysis of the affected members.

A non-consanguineous Japanese couple was referred to our hospital (Fig. 1a, II-2 and II-3) for third pregnancy management. They had previously experienced two consecutive stillbirths with fetal malformations (Fig. 1a, III-1

\footnotetext{
Correspondence: Kenichiro Hata (hata-k@ncchd.go.jp)

'Department of Obstetrics and Gynecology, The Jikei University School of

Medicine, 3-25-8, Nishi-Shinbashi, Minato-ku, Tokyo 105-8461, Japan

2Department of Maternal-Fetal Biology, National Research Institute for Child

Health and Development, 2-10-1, Okura, Setagaya-ku, Tokyo 157-8535, Japan

Full list of author information is available at the end of the article
}

and III-2). The mother (II-3) had been born prematurely with intrauterine growth restriction and presented at birth with a cleft lip, nasolacrimal duct obstruction, bilateral curled selvages, and severe hearing loss in her right ear. We also detected a previously undiagnosed skin defect scar on the right side of her neck. Both stillborn fetuses had bilateral polycystic kidneys, and the pregnancies were severely oligohydramnios. The second offspring (Fig. 1a, III-2) was male and also had a cleft lip and palate.

During the second trimester of the third pregnancy, sonographic fetal screening detected only moderate fetal growth restriction. However, due to tocolysis failure, a male infant (Fig. 1a, III-3) was born at 32 weeks of gestation and subsequently admitted to the neonatal intensive care unit due to respiratory distress syndrome and low birth weight (1399 g). Detailed clinical examination revealed cutaneous defects and erythema on the left side of his cervical and periauricular regions, wide nasal tips, low-set ears, and micrognathia. Laryngomalacia and tracheal dysplasia were also suspected. Following ophthalmologic examination, he was diagnosed with bilateral colobomas, left microphthalmia, and nasolacrimal duct obstruction. His karyotype was $46, \mathrm{XY}$. The mother had a fourth pregnancy one year after the birth of III-3. At 10weeks of gestation, the fetus (Fig. 1a, III-4) was diagnosed by ultrasound examination with acrania. Following 
a

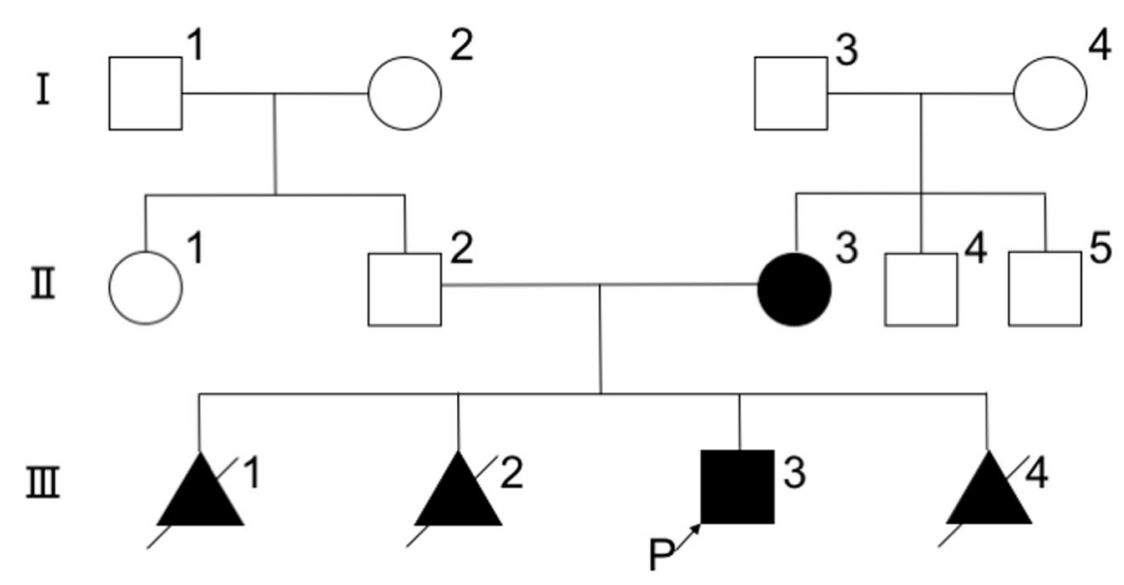

b

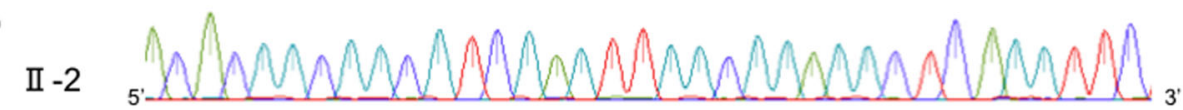

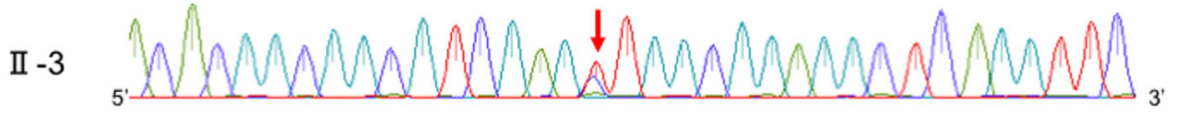

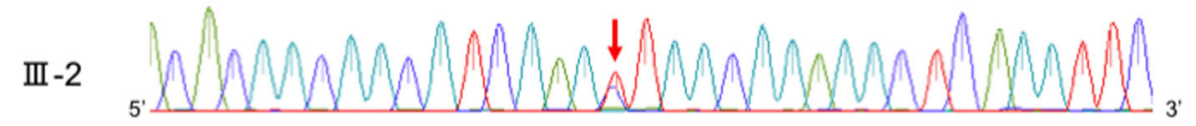

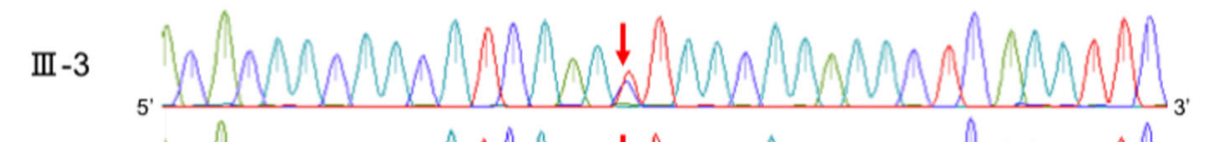

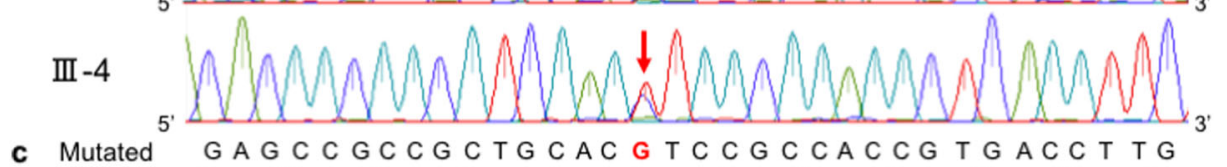

c Mutated GAGCCGCCGCTGCACGTCCGCCACCG TGACCT TG

\begin{tabular}{|c|c|c|c|c|c|c|c|c|c|c|}
\hline \hline$L$ & $R$ & $R$ & $Q$ & $V$ & $D$ & $A$ & $V$ & $T$ & $V$ & $K$ \\
\hline
\end{tabular}

Reference GAGCCGCCGCTGCACT T C CGCCACCG TGACCT TG

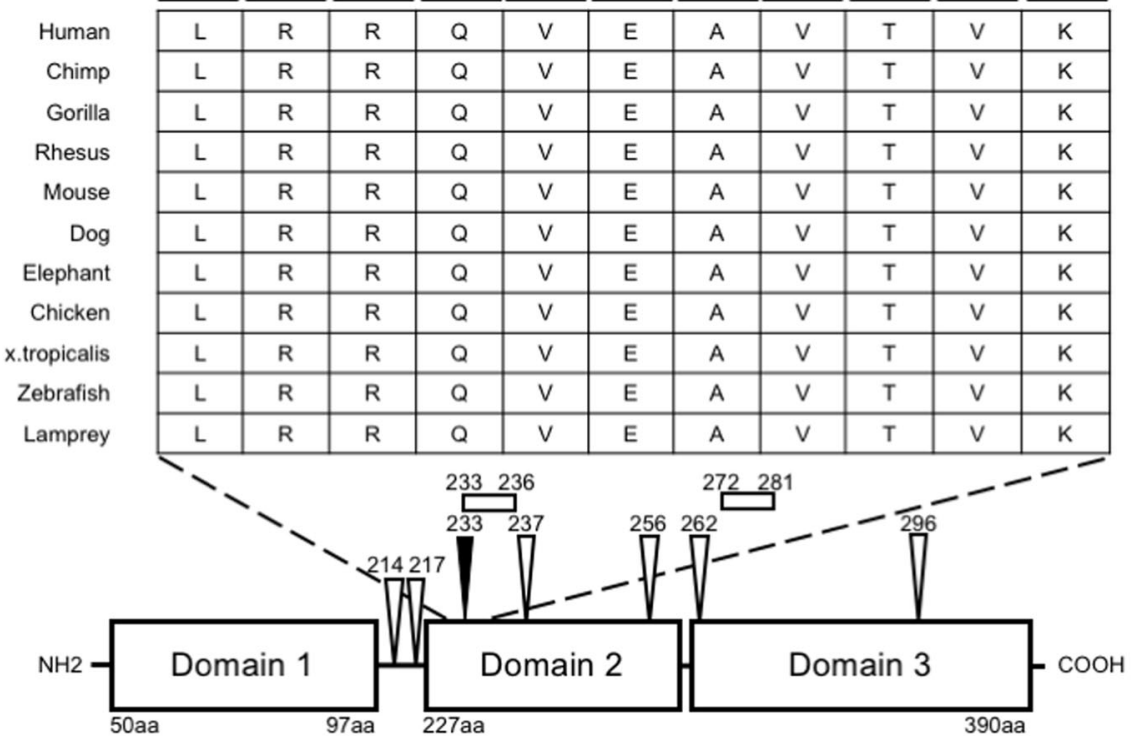

Fig. 1 (See legend on next page.) 
(see figure on previous page)

Fig. 1 A novel missense TFAP2A mutation identified in a Japanese family. a A family pedigree of the Japanese family with Branchio-oculo-facial syndrome. $\mathbf{b}$ Sequence confirmation of a TFAP2A mutation in study members II-2, II-3, III-2, III-3, and III-4. Each nucleotide is shown in a different color: adenine (A), green; cytosine (C), aqua; guanine (G), purple; and thymine (T), red. Red arrows indicate the novel mutation we detected. c Amino-acid alignment proximal to the novel TFAP2A mutation in various species. The region around E233D is evolutionarily conserved. $\mathbf{d}$ Protein domains and reported pathogenic amino-acid substitutions of TFAP2A. The E233D substitution (black arrowhead) is positioned near pathogenic amino-acid substitutions (white bars and white arrowheads) registered at ClinVar (https://www.ncbi.nlm.nih.gov/clinvar/). Domain 1, proline- and glutamine-rich domain for transactivation; Domain 2, basic domain of DNA-binding region; and Domain 3, Helix-span-helix domain mediates dimerization and sitespecific DNA binding

Table 1 Summary of clinical features of the patients in this family

\begin{tabular}{|c|c|c|c|c|c|}
\hline & II-3 & III-1 & III-2 & III-3 & III-4 \\
\hline Age & 35-years old & 21-weeks of gestation & 18-weeks of gestation & One-year-old & 10-weeks of gestation \\
\hline Karyotype & $46, X X$ & Unknown & $46, X X$ & $46, X Y$ & $46, X X$ \\
\hline Phenotypic severity & Relatively mild & Severe & Severe & Relatively mild & Severe \\
\hline Preterm birth & + & Unknown & Unknown & + & Unknown \\
\hline Acrania & - & - & - & - & + \\
\hline Microphthalmia & - & Unknown & Unknown & + & Unknown \\
\hline Optic nerve coloboma & - & Unknown & Unknown & + & Unknown \\
\hline Cleft lip and palate & + & Unknown & + & - & Unknown \\
\hline Nasolacrimal duct obstruction & + & Unknown & Unknown & + & Unknown \\
\hline Low-set ears & + & Unknown & + & + & Unknown \\
\hline Hearing loss & + & Unknown & Unknown & - & Unknown \\
\hline Facial skin defect & + & Unknown & - & + & Unknown \\
\hline Micrognathia & + & Unknown & - & + & Unknown \\
\hline Polycystic kidney & - & + & + & - & Unknown \\
\hline Severe potter syndrome & - & + & + & - & Unknown \\
\hline Intellectual disability & - & Unknown & Unknown & - & Unknown \\
\hline
\end{tabular}

genetic counseling, she and her partner chose to terminate the pregnancy.

Because similar malformations were detected in family members II-3, III-2, and III-3, an inherited anomalad phenotypically closest to $\mathrm{BOFS}^{1}$ was suspected. However, because a discordance in the severity of malformations was observed among family members and fetuses (Table 1), detailed molecular genetic analyses were performed. The study was approved by the Institutional Review Boards (IRBs) of the National Center for Child Health and Development and the Jikei University School of Medicine (IRB number: 234 and IRB number: 27-060 (7945), respectively). Written informed consent was obtained from the parents.

DNA was extracted from the peripheral blood samples of II-2 and II-3 using a QIAamp DNA Blood Midi Kit (Qiagen, Venlo, Netherlands) following the manufacturer's instructions. DNA was extracted from the formalin-fixed placental chorionic villi of III-2, the umbilical cord of III-3, and an arm specimen of fetus III-4 using conventional phenol/chloroform/isoamyl alcohol methods. Genome-wide single-nucleotide polymorphism (SNP) array analysis of the DNA samples was performed using the HumanCytoSNP-12 DNA Analysis BeadChip Kit (Illumina, San Diego, CA, USA) according to the manufacturer's protocols. The SNP arrays were scanned using the Illumina iScan system, and the data were analyzed with Illumina's KaryoStudio (version 1.4) using the default cnvPartition algorithm settings (version 3.0.7, Illumina).

Sequencing was performed on the II-2, II-3, and III-3 DNA samples. A whole-exome library of each sample was prepared using the Agilent SureSelect v6 Capture Kit (Agilent Technologies, Santa Clara, CA, USA) following 
the manufacturer's protocol. The libraries were sequenced on an Illumina HiSeq2500 in the 101-bp paired-end mode. Sequence reads were mapped and aligned to the reference genome sequence hs37d5 using our pipeline including Grepwalk (http://epigenetics. nrichd.ncchd.go.jp/grepwalk/), Cutadapt-1.7.1 (https:// cutadapt.readthedocs.io/en/stable/), BWA MEM (http:// bio-bwa.sourceforge.net/), SAMtools 1.3 (http://samtools. sourceforge.net/), Picard 2.1.1 (http://picard.sourceforge. net/), and Genome Analysis Toolkit 3.5 (https://software. broadinstitute.org/gatk/). Multi-sample calling of singlenucleotide variations and short indels was performed with the RefSeq gene database in combination with eight in-house control datasets. The variant frequencies were compared to reference sets from the 1000 Genomes Project database (1KGP) (http://www. internationalgenome.org), the Human Genome Variation Database (HGVD; http://www.hgvd.genome.med.kyoto-u. ac.jp), and the integrated Japanese Genome Variation Database (iJGVD; https://ijgvd.megabank.tohoku.ac.jp). To identify candidate pathogenic variants in the III-3 dataset, we extracted mutations at exons and splice sites with an allele frequency threshold of $<0.2$ or $>0.8$ based on $1 \mathrm{KGP}$ data and to filter Japanese common variant, that of $<0.1$ or $>0.9$ based on the Japanese data from the HGVD, iJGVD and Japanese in Tokyo (JPT) data from 1KGP, respectively. We also selected candidate variants that had a read coverage threshold $>8$ and that were not detected in more than two individuals in in-house control datasets.

Genome-wide SNP array analyses showed no mosaicism or chimerism signal aberrations. A copy-number loss was detected at 10q21.3 in the III-2 and III-3 datasets and at 7q11.23 in the III-2 dataset. However, these losses were inherited paternally and did not contain any reported disease-related genes (Supplementary Table S1). Wholeexome sequencing identified 1034 variants as potential pathogenic nucleotide alterations. Of these variants, 319 were common to both the mother and the newborn (Supplementary Table S2), and one was a missense point mutation of a thymine-to-guanine transition at nucleotide position 699 within exon 4 of TFAP2A. This change results in a predicted substitution of glutamine 233 with aspartic acid (E233D). To the best of our knowledge, this variant has not been reported previously in dbSNP; 1KGP; HGVD; iJGVD; Exome Variant Server of the National Heart, Lung, and Blood Institution Grand Opportunity Exome Sequencing Project (http://evs.gs.washington.edu/ EVS/); Human Genome Mutation Database ${ }^{6}$; and Exome Aggregation Consortium (http://exac.broadinstitute.org) databases. None of the other detected candidate variants were suspected to be involved in the development of the observed phenotypes, including renal dysfunctions or malformations. To confirm the TFAP2A mutation and to determine whether III- 2 and III- 4 shared this mutation, we performed dye-terminator direct sequencing. The results demonstrated that the thymine-to-guanine substitution at nucleotide 699 within exon 4 of TFAP2A was shared with all of the offspring that we analyzed (Fig. 1b). These findings provided genetic support for the clinical diagnosis of BOFS.

We identified a novel point mutation located in a highly conserved region of exon 4 of TFAP2A in a Japanese family. The predicted result is an E233D amino-acid substitution in the basic DNA-binding domain of TFAP2A (Fig. 1b-d) ${ }^{7}$. As depicted in Fig. 1d, multiple pathogenic variants of TFAP2A have been reported in the vicinity of this mutation. Furthermore, some mutations at this basic DNA-binding domain have also been reported to cause a drastically reduced AP2-alpha transactivation ${ }^{8}$. Additionally, in silico analyses predicted that the E233D amino-acid substitution may be pathogenic (polyphen-2 HDIV, 0.99; polyphen-2 HVAR, 0.994; SIFT, 0.002) $)^{9,10}$. These observations suggested that this region may be significant for TFAP2A functions in BOFS development. We propose that the novel TFAP2A mutation identified in this study is a potential pathogenic mutation that contributed to or directly caused the development of BOFS in the described Japanese family.

Reported BOFS-associated malformations are diverse $^{1,11}$. However, only TFAP2A alterations have been identified as underlying genetic causes. The family in our study exhibited similar malformations, but the mutations had differing severities. Genetic analysis demonstrated low possibilities of cell mosaicism for each affected individual; however, other mutations may have caused the lethal phenotypes in III-1, III-2, and III-4. Similar pedigrees with intrafamilial phenotypic variety have been described, indicating that BOFS genotype-phenotype correlations may not be very strict within a family and may be due to unknown genetic factors ${ }^{11,12}$. Additional analyses of multiple BOFS families will be required to clarify other genetic factors involved in the development of BOFS phenotypic varieties.

In conclusion, we identified a novel point mutation in TFAP2A in a highly conserved region of the basic DNAbinding domain. The predicted result is a glutamate to aspartic acid substitution at position 233, which may be responsible for or at least a contributor to the development of BOFS. Although intrafamilial phenotype severity was also observed in this pedigree, no other causative genetic aberrations were detected. Future advance in phenotypic and genetic detection method will help identify additional genetic causes underlying the observed phenotypic variety in patients with BOFS.

\section{HGV Database}

The relevant data from this Data Report are hosted at the Human Genome Variation Database at https://doi.org/10.6084/m9.figshare.hgv.1936. DDBJ accession number: LC336807 


\section{Acknowledgements}

We are deeply grateful to the patients and their family members for participating in this study. This work was supported by a grant from the National Center for Child Health and Development (NCCHD 26-13).

\section{Author details}

'Department of Obstetrics and Gynecology, The Jikei University School of Medicine, 3-25-8, Nishi-Shinbashi, Minato-ku, Tokyo 105-8461, Japan.

${ }^{2}$ Department of Maternal-Fetal Biology, National Research Institute for Child Health and Development, 2-10-1, Okura, Setagaya-ku, Tokyo 157-8535, Japan. ${ }^{3}$ Department of Pediatrics, The Jikei University School of Medicine, 3-25-8, Nishi-Shinbashi, Minato-ku, Tokyo 105-8461, Japan

\section{Conflict of interest}

The authors declare that they have no conflict of interest.

\section{Publisher's note}

Springer Nature remains neutral with regard to jurisdictional claims in published maps and institutional affiliations.

Supplementary information is available for this paper at https://doi.org/ 10.1038/s41439-018-0004-z.

Received: 19 December 2017 Revised: 18 February 2018 Accepted: 9 March 2018.

Published online: 10 May 2018

\section{References}

1. Lin, A. E. et al. Further delineation of the branchio-oculo-facial syndrome. Am J. Med. Genet. 56, $42-59$ (1995).
2. Milunsky, J. M. et al. TFAP2A mutations result in branchio-oculo-facial syndrome. Am. J. Hum. Genet. 82, 1171-1177 (2008).

3. Bakrania, P. et al. Mutations in BMP4 cause eye, brain, and digit developmental anomalies: overlap between the BMP4 and hedgehog signaling pathways. Am. J. Hum. Genet. 82, 304-319 (2008).

4. Gestri, G. et al. Reduced TFAP2A function causes variable optic fissure closure and retinal defects and sensitizes eye development to mutations in other morphogenetic regulators. Hum. Genet. 126, 791-803 (2009).

5. Budirahardja, Y., Tan, P. Y., Doan, T., Weisdepp, P. \& Zaidel-Bar, R. The AP-2 transcription factor APTF-2 is required for neuroblast and epidermal morphogenesis in Caenorhabditis elegans embryogenesis. PLoS Genet. 12, e1006048 (2016).

6. Stenson, P. D. et al. The Human Gene Mutation Database: building a comprehensive mutation repository for clinical and molecular genetics, diagnostic testing and personalized genomic medicine. Hum. Genet. 133, 1-9 (2014).

7. Bauer, R. et al. The genomic structure of the human AP-2 transcription factor. Nucl. Acids Res. 22, 1413-1420 (1994).

8. Adzhubei, I. A. et al. A method and server for predicting damaging missense mutations. Nat. Methods 7, 248-249 (2010).

9. Li, H., Sheridan, R. \& Williams, T. Analysis of TFAP2A mutations in Branchio-oculo-facial Syndrome indicates functional complexity within the AP-2alpha DNA-binding domain. Hum. Mol. Genet. 22, 3195-3206 (2013).

10. Ng, P. C. \& Henikoff, S. Predicting deleterious amino acid substitutions. Genome Res. 11, 863-874 (2001)

11. Meshcheryakova, T. I. et al. A clinical and molecular analysis of branchio-oculofacial syndrome patients in Russia revealed new mutations in TFAP2A. Ann Hum. Genet. 79, 148-152 (2015).

12. Titheradge, H. L., Patel, C. \& Ragge, N. K. Branchio-oculo-facial syndrome: a three generational family with markedly variable phenotype including neonatal lethality. Clin. Dysmorphol. 24, 13-16 (2015). 